ROSA, M.A. et al. Anatomia da cloaca de algumas espécies de Pleurodira (Reptilia:

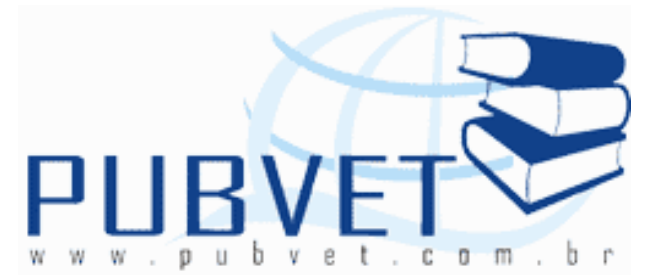

PUBVET, Publicações em Medicina Veterinária e Zootecnia.

\title{
Anatomia da cloaca de algumas espécies de Pleurodira (Reptilia: Testudines)
}

\author{
Marcelo Alcântara Rosa ${ }^{1}$, André Luiz Quagliatto Santos ${ }^{2}$, Lorena Tannús \\ Menezes ${ }^{1}$, Arthur Paulino Sanzo Kaminishi ${ }^{1}$, Tatiana Grillo Leonardo ${ }^{1}$, Natália \\ Siqueira D'Aparecida ${ }^{3}$
}

Laboratório de Ensino e Pesquisas em Animais Silvestres - LAPAS, FAMEV/UFU, e-mail: quagliatto@famev.ufu.br 1. Mestrandos. 2. Docente. 3. Graduanda.

\section{Resumo}

O estudo proposto investiga as disposições das aberturas dos ureteres, da uretra e dos ovidutos nas fêmeas e dos ductos deferentes nos machos e o arranjo dos compartimentos da cloaca, em quatro espécies das famílias Podocnemididae e Chelidae. As cloacas, estudadas anatomicamente, são formadas por três compartimentos: coprodeu, urodeu e proctodeu. No entanto, nenhuma delas está conectada aos ureteres, pois estes desembocam diretamente no urodeu. Nas espécies pesquisadas do gênero Podocnemis, existe uma membrana que separa o coprodeu do urodeu, mas que não está presente nos espécimes da família Chelidae, utilizados nesta pesquisa. Em Podocnemis expansa, Podocnemis unifilis e Chelus fimbriatus, a uretra se insere cranioventralmente no urodeu, mas, em Phrynops geoffroanus, a uretra conecta-se com o urodeu em posição vertical e ventral. Nos machos de Podocnemis, os ductos deferentes desembocam em papilas intramurais e 
ROSA, M.A. et al. Anatomia da cloaca de algumas espécies de Pleurodira (Reptilia: Testudines). PUBVET, Londrina, V. 5, N. 11, Ed. 158, Art. 1070, 2011.

caudalmente aos óstios dos ureteres. Nos machos de $P$. geoffroanus e de $C$. fimbriatus e em todas as fêmeas estudadas, os ductos dos aparelhos reprodutores e os ureteres abrem-se em um seio urogenital. Nas fêmeas de $P$. geoffroanus e de C. fimbriatus, em estágio de reprodução, os ovidutos projetam-se para o urodeu e formam um par de papilas de contornos arredondados, com uma abertura ventral. Nas espécies do gênero Podocnemis, o urodeu comunica-se com o proctodeu por meio de uma fenda. Estão presentes no proctodeu duas bolsas cloacais. A passagem do coprodeu para 0 proctodeu ocorre caudodorsalmente à prega urogenital.

Palavras-chave: cloaca, bolsa cloacal, coprodeu, urodeu, proctodeu, Testudines e Pleurodira.

\section{Anatomy of the Cloaca of some Pleurodira species (Reptilia: Testudines)}

\section{Abstract}

The proposed study investigates the provisions of the openings of the ureters, urethra, oviducts of the female and of the deferens ducts in the males, and the arrangement of the cloacae compartments in four species of the Podocnemididae and Chelidae family. The cloacae, anatomically studied, are composed of three compartments: coprodeu, urodeu, proctodeu. However, none of them is attached to the ureters, because they culminate directly in urodeu. In the species of the genus Podocnemis surveyed, there is a membrane that separates the coprodeu do urodeu, but that is not present in the specimens of the family Chelidae used in this research. In Podocnemis expansa, Podocnemis unifilis and Chelus fimbriatus, the urethra inserts cranioventrally in the urodeu, but in Phrynops geoffroanus, the urethra connects to the urodeu in upright and ventral position. In males of Podocnemis, the deferens ducts culminates in intramurals papillae and caudally of the ostia of the ureters. In males of $P$. geoffroanus and $C$. fimbriatus and in all females studied, the ducts of the reproductive tract and the ureters open 
ROSA, M.A. et al. Anatomia da cloaca de algumas espécies de Pleurodira (Reptilia: Testudines). PUBVET, Londrina, V. 5, N. 11, Ed. 158, Art. 1070, 2011.

into a urogenital sinus. In females of $P$. geoffroanus and $C$. fimbriatus, in reproduction stage, the oviducts are projects to the urodeu and form a pair of papillae rounded contours, with a ventral opening. In the species of the genus Podocnemis, the urodeu communicates with proctodeu through a slit. In proctodeu are present two cloacae bags. The passage of the coprodeu for the proctodeu occurs caudodorsally to the urogenital fold.

Keywords: cloaca, cloacal bag, coprodeu, urodeu, proctodeu, Testudines, Pleurodira.

\section{INTRODUÇÃO}

Cágados possuem a pelve fundida ao casco e flexionam o pescoço lateralmente para escondê-lo entre o plastrão e a carapaça. Pertencem à ordem Testudines e à subordem Pleurodira (LEGLER e GEORGES, 1993) e são encontrados apenas no hemisfério sul: África, Austrália e América do Sul (PETERSON e GREENSHIELDS, 2001). Pough, Janis e Heiser (2003) separam as espécies de cágados em três famílias: Chelidae, Pelomedusidae e Podocnemididae.

Podocnemis expansa (Schweigger, 1812), conhecida popularmente por tartaruga-da-Amazônia e Podocnemis unifilis Troshcel, 1848 ou tracajá são espécies dos rios da bacia Amazônica, preferencialmente aquáticas e que saem da água para desovar e para aquecer ao sol. Há certa separação de hábitat entre as duas espécies, os adultos de $P$. expansa habitam grandes rios enquanto que os de $P$. unifilis são encontrados em seus tributários. Durante a estação de chuvas, quando os rios enchem, os indivíduos e $P$. unifilis buscam refúgio das águas rápidas e turbulentas e migram para as florestas inundadas e lagoas com águas mais calmas (PRITCHARD e TREBBAU, 1984).

Phrynops geoffroanus (Schweigger, 1812), cágado de barbicha, e Chelus fimbriatus (Schneider, 1783) ou matamatá são espécies da família Chelidae. $P$. geoffroanus é encontrado em rios, lagos e córregos de diversos ecossistemas da região neotropical (GUIX et al., 1989). Possui um par de barbelas brancas com extremidades pretas no queixo e são vistos com freqüência nas margens 
ROSA, M.A. et al. Anatomia da cloaca de algumas espécies de Pleurodira (Reptilia: Testudines). PUBVET, Londrina, V. 5, N. 11, Ed. 158, Art. 1070, 2011.

e sobre troncos de rios, remansos e lagoas nas horas de sol (PRITCHARD e TREBBAU, 1984). Chelus fimbriatus tem casco e cabeça largos e achatados e numerosas abas de pele que servem para a percepção de vibrações na água, provocadas pelas presas e para a fixação de algas. O matamatá não possui o bico córneo que outros quelônios utilizam para capturar a presa ou arrancar pedaços de plantas e é encontrado nos rios no norte da América do Sul (MÉTRAILLER, 2003; POUGH, JANIS e HEISER, 2003).

Gomes (1994) salienta que no Brasil há falta de tradição na pesquisa morfológica de répteis. Realmente, estudos anatômicos em espécies de testudines brasileiras são escassos, porém são de grande importância para várias áreas de pesquisa, inclui-se entre elas a sistemática e a preservação (MALVÁSIO, 1996).

A cloaca é uma bolsa na extremidade posterior do corpo que se comunica com o exterior e constitui o caminho comum para os produtos dos tratos digestivo, genital e urinário (ROMER e PARSONS, 1985, HILDEBRAND, 1995) e divide-se em três compartimentos: coprodeu, urodeu e proctodeu.

Coprodeu é o compartimento mais cranial e recebe os restos fecais do reto. O urodeu contém as aberturas dos ureteres, da bexiga urinária, dos ovidutos nas fêmeas e dos ductos deferentes nos machos e o proctodeu, maior parte da cloaca, tem posição caudal e serve como reservatório para a eliminação fecal e urinária (BENNETT, 2006). Esses compartimentos são delimitados nas aves, respectivamente, por dobras denominadas retrocoprodeal, coprourodeal e uroproctodeal (OLIVEIRA et al., 2004). Nas paredes laterais do proctodeu, de alguns testudines, existem expansões denominadas bolsas cloacais ou bexigas acessórias (JORGENSEN, 1998; PETERSON e GREENSHIELDS, 2001). Em uma revisão sobre as prováveis funções dessas bolsas, Peterson e Greenshields (2001) concluíram que para os pleurodira a função seria a de respiração aquática, como uma brânquia.

O objetivo deste estudo foi investigar as disposições das aberturas dos ureteres, da uretra e dos ductos dos aparelhos reprodutor masculino e 
ROSA, M.A. et al. Anatomia da cloaca de algumas espécies de Pleurodira (Reptilia: Testudines). PUBVET, Londrina, V. 5, N. 11, Ed. 158, Art. 1070, 2011.

feminino, bem como o arranjo dos compartimentos da cloaca, em quatro espécies de cágados das famílias Podocnemididae e Chelidae.

\section{MATERIAL E MÉTODOS}

Os oito exemplares utilizados neste trabalho encontravam-se fixados em solução de formol a $10 \%$ e foram fornecidos pelo Laboratório de Ensino e Pesquisa em Animais Silvestres (LAPAS) da Faculdade de Medicina Veterinária (FAMEV) da Universidade Federal de Uberlândia (UFU), obtidos com licenças 066/2004, 117/2005, 120/2005 e 032/2006 - IBAMA/RAN. A retirada do plastrão foi feita com serra mármore, tesoura, bisturi e pinças anatômicas. Em seguida realizou-se observação geral da disposição das vísceras e retirou-se o trato gastrintestinal, o fígado e o coração. Sem o trato gastrintestinal, o sistema urogenital foi individualizado e retirado da carcaça, para ser fotografado e analisado.

Abriu-se a cloaca, inicialmente, pela região dorsal do coprodeu, através do segmento final do intestino grosso. Seccionou-se o proctodeu e as bolsas cloacais e, finalmente, abriu-se o urodeu e a uretra até atingir à bexiga urinária. Introduziram-se agulhas hipodérmicas nos ureteres, na uretra, nos ovidutos e nos ductos deferentes para verificar as aberturas dos seus óstios no urodeu. Em seguida todas as peças foram fotografadas, analisadas e imersas em álcool $70^{\circ} \mathrm{GL}$.

\section{RESULTADOS}

A cloaca dos pleurodiras, estudados anatomicamente, é formada por três compartimentos, coprodeu, urodeu e proctodeu e integra o sistema urogenital e o sistema digestório (figura 1). Abrem-se, na cloaca, a porção final do colorreto, a uretra, os ureteres, os ovidutos (fêmea) e os ductos deferentes (machos). No proctodeu encontram-se duas dilatações da parede, as bolsas cloacais (figura 1 b). 
ROSA, M.A. et al. Anatomia da cloaca de algumas espécies de Pleurodira (Reptilia:

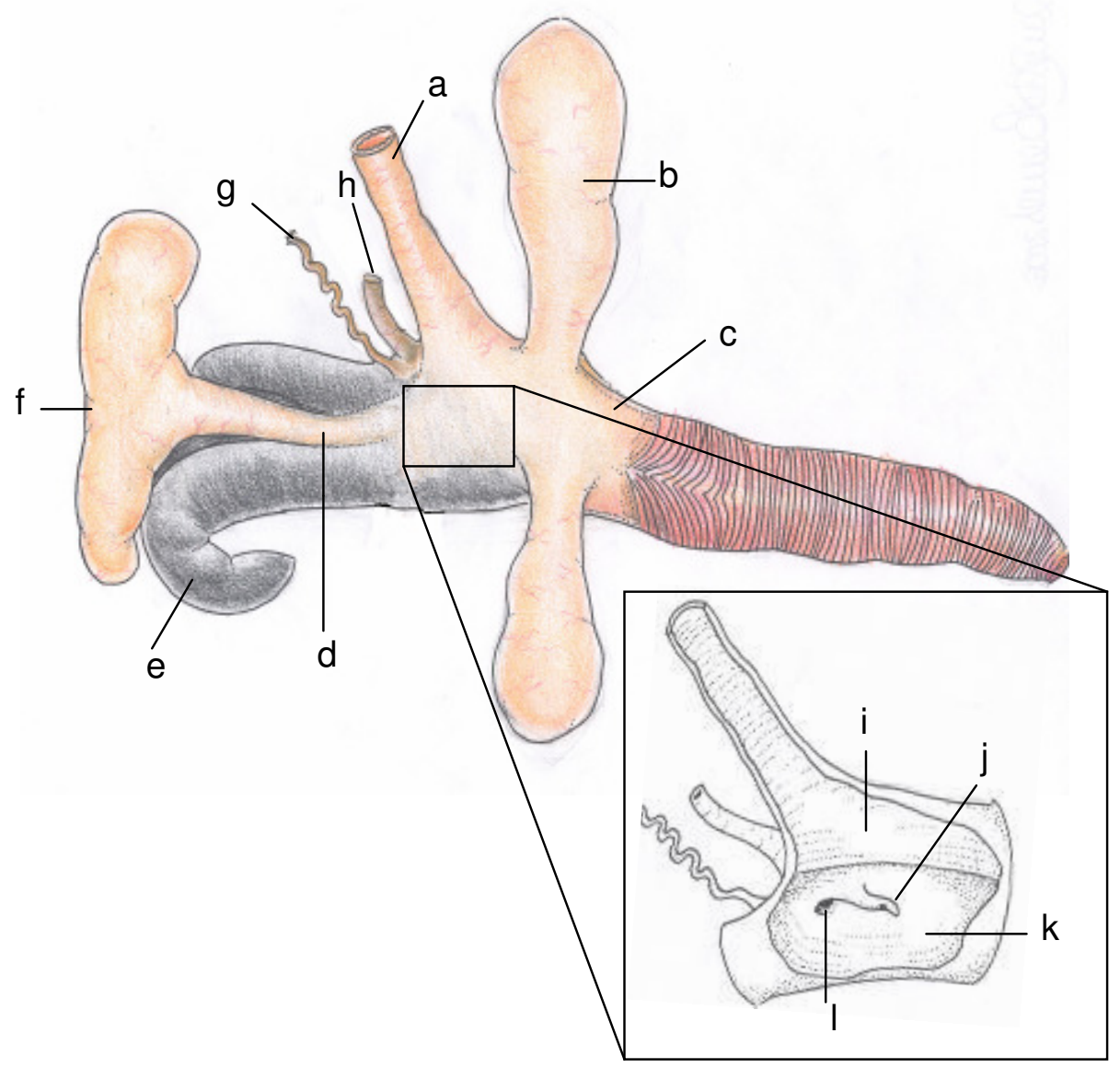

Figura 1: Vista lateral da cloaca de Podocnemis expansa. a: colorreto; b: bolsa cloacal; c: proctodeu; d: uretra; e: pênis; f: bexiga urinária; g: ducto deferente; $\mathbf{h}$ : ureter; $\mathbf{i}$ : coprodeu; $\mathbf{j}$ : papila genital; $\mathbf{k}$ : urodeu e I: óstio ureteral.

Coprodeu (figura 1i) é o compartimento cranial e dorsal da cloaca, comunica-se dorsalmente com o colorreto (figura 1a), cuja mucosa é pregueada longitudinalmente. Nas espécies do gênero Podocnemis, observa-se uma espessa membrana que separa o coprodeu do urodeu e que está ausente nos cágados da família Chelidae utilizados nesta pesquisa.

O urodeu (figura $1 \mathrm{k}$ ) é o compartimento cranial e ventral da cloaca no qual se inserem a uretra (figura 1d), os ureteres, os ovidutos nas fêmeas e os ductos deferentes nos machos, sendo que, nos podocnemidideos, ocorre inversão de posição entre os ureteres e os ductos genitais quanto ao percurso 
ROSA, M.A. et al. Anatomia da cloaca de algumas espécies de Pleurodira (Reptilia: Testudines). PUBVET, Londrina, V. 5, N. 11, Ed. 158, Art. 1070, 2011.

desses tratos na parede da cloaca. Em $P$. expansa, $P$. unifilis e C. fimbriatus, a uretra se insere cranioventralmente no urodeu, mas, em $P$. geoffroanus, a uretra conecta-se com o urodeu em posição vertical e ventral (figura $2 b$ ).

Ductos deferentes e ovidutos inserem-se lateralmente na parede do urodeu e cranialmente aos ureteres. Nos machos de Podocnemis, os ductos deferentes (figura $1 \mathrm{~g}$ ) desembocam em papilas genitais (figura $1 \mathrm{j}$ ) e caudalmente aos óstios ureterais (figura 11 ). Nos machos de $P$. geoffroanus e de C. fimbriatus e em todas as fêmeas estudadas, os ductos dos aparelhos reprodutores e os ureteres abrem-se em um seio urogenital. Nas fêmeas de $P$. geoffroanus e de $C$. fimbriatus, em estágio de reprodução, os ovidutos (figura 2d) projetam-se para $o$ urodeu e formam projeções de contornos arredondados, com abertura ventral.

Dorsolateralmente ao proctodeu (figura $1 \mathrm{c}$ ) inserem-se as bolsas cloacais e, na parede ventral - no caso dos machos - está presente o pênis. O urodeu comunica-se com o proctodeu por meio de uma fenda. A passagem do coprodeu para o proctodeu ocorre caudodorsalmente à prega urogenital. 
ROSA, M.A. et al. Anatomia da cloaca de algumas espécies de Pleurodira (Reptilia: Testudines). PUBVET, Londrina, V. 5, N. 11, Ed. 158, Art. 1070, 2011.

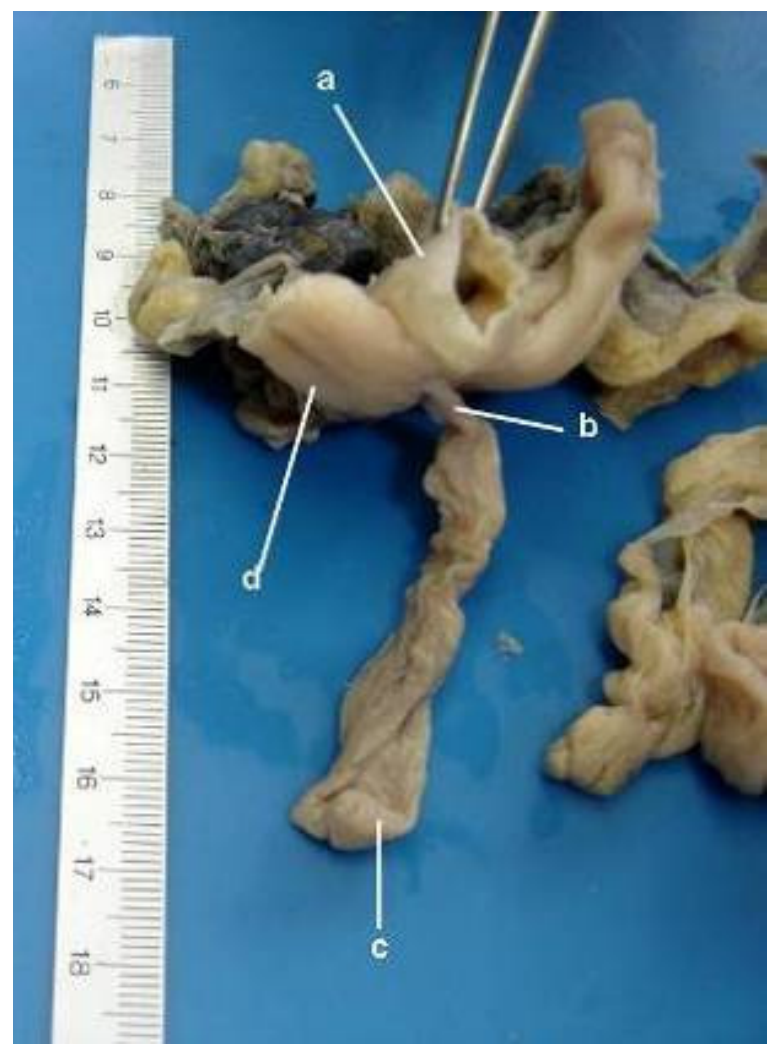

Figura 2: Fotografia da Cloaca de Phrynops geoffroanus fêmea. a: colorreto; b: uretra; c: bexiga urinária; d: oviduto.

\section{DISCUSSÃO}

Os compartimentos cloacais, coprodeu, urodeu e proctodeu, pesquisados durante a dissecação, são semelhantes aos descritos em aves por King (1981); Oliveira et al. (2004). Estudos, feitos por King (1981), em cloacas de aves, descrevem estruturas membranosas que individualizam as três regiões da cloaca, como nas espécies de podocnemis dissecadas nesta pesquisa, porém com diferenças na espessura dessa estrutura. Dulzetto (1967) afirma que, em Testudo $s p$, não existe delimitação entre o coprodeu e o urodeu. Membranas também estão ausentes na cloaca das espécies da família Chelidae pesquisadas. A denominação das membranas de delimitação dos 
ROSA, M.A. et al. Anatomia da cloaca de algumas espécies de Pleurodira (Reptilia: Testudines). PUBVET, Londrina, V. 5, N. 11, Ed. 158, Art. 1070, 2011.

compartimentos da cloaca mencionadas por Oliveira et al. (2004), para aves, não encontram correspondência nas investigações em testudines.

Messer (1947) em répteis em geral e Faria (2003) em Geochelone carbonaria descreveram que os ureteres inserem-se na bexiga urinária. Já, em Caiman crocodilus yacare, Oliveira et al. (2004) relatam a finalização dos ureteres no coprodeu, diferentemente dos achados em $P$. expansa, $P$. unifilis, $P$ geoffroanus e $C$. fimbriatus. Nessas espécies, verificou-se que os ureteres terminam lateralmente no urodeu e caudalmente aos ovidutos nas fêmeas e aos ductos deferentes nos machos e abrem-se, separadamente, no interior da cloaca, no seio urogenital e não estão conectados diretamente com a bexiga urinária, conforme relatos de Weichert (1951), Montagna (1959) e Dulzetto (1967). Em Geochelone carbonaria (Faria, 2003), bem como em machos de $P$. expansa e $P$. unifilis, os ductos deferentes desembocam em papilas genitais, porém com posicionamentos diferentes no urodeu, o que está de acordo com Hyman (1957). Não foram encontrados, na literatura consultada, relatos de que os ovidutos de fêmeas de $P$. geoffroanus e $C$. fimbriatus, em estágio de reprodução, projetam-se para o interior do urodeu e suas aberturas dirigem-se ventralmente.

Dentro da cloaca, encontram-se as aberturas da bexiga urinária - uretra e das bexigas acessórias, Em $P$. expansa, $P$. unifilis e $C$. fimbriatus a uretra insere-se cranioventralmente no urodeu e as bolsas cloacais estão ligadas dorsolateralmente às paredes do proctodeu, conforme afirmaram Hyman (1957), Montagna (1959), Malvásio (1996), Jorgensen (1998), Peterson e Greenshields (2001) e Mallmann-Franco (2002). Por outro lado, a uretra em $P . g e o f f r o a n u s$ insere-se ventralmente no urodeu.

\section{CONCLUSÃO}

Cloaca é uma estrutura que apresenta variações nos seus compartimentos e nas disposições anatômicas da uretra, dos ureteres e dos ductos deferentes nos machos, entre as espécies de uma mesma família da subordem Pleurodira. 
ROSA, M.A. et al. Anatomia da cloaca de algumas espécies de Pleurodira (Reptilia:

Testudines). PUBVET, Londrina, V. 5, N. 11, Ed. 158, Art. 1070, 2011.

\section{REFERÊNCIAS}

BENNETT, R. A. Cloacal Prolapse. In: MADER, D.R. (Ed.). Reptile medicine and surgery, Philadelphia: Saunders Elsevier, 2006, cap 37, 2ed, p. 355.

DULZETTO, F. Anatomia comparada dei vertebrati. Bologna: Edizioni Calderini, 1967.

FARIA, T. N. Topografia e morfologia do sistema urinário de jabuti Geochelone carbonaria (Spix, 1824). São Paulo: USP, 2003. 86p. Tese. (Doutorado em Animais Domésticos e Silvestres) - Programa de Pós-graduação em Animais Domésticos e Silvestres, Faculdade de Medicina Veterinária e Zootecnia, Universidade de São Paulo, São Paulo, 2003.

GOMES, N. Linhas gerais de pesquisa em herpetologia. In: NASCIMENTO, L. B.; BERNARDES, A. T.; COTTA, G. A. Herpetologia no Brasil. PUC-MG/Fundação, Belo Horizonte: Ezequiel Dias, 1994, p. 128-129.

GUIX, J. C.; SALVATTI, M.; PERONI, M. A; LIMA-VERDE, J. S. Aspectos da reprodução de Phrynops geoffroanus em cativeiro (Testudine, Chelidae). Grupo Estudos Ecológicos/ Série Documentos, São Paulo, v.1, p.1-19, Set. 1989.

HILDEBRAND, M. Análise das estruturas dos vertebrados. São Paulo: Atheneu, 1995.

HYMAN, L. M. Comparative vertebrate. Chicago: The University of Chicago Press, 1957.

JORGENSEN, C. B. Role of urinary and cloacal bladders in chelonian water economy: historical and comparative perspectives. Biological Review, London, v.73, p. 347-366, Mar. 1998.

KING, A. S. Cloaca. In: KING, A. S.; MCLELLAND, J. (Eds.), Form and function in birds, London: Academic Press, 1981, v. 2, cap 2, p. 63-105.

LEGLER, J. M.; GEORGES, A. Family Chelidae. In: GLASBY, C. J.; ROSS, G. J. B.; BEESLEY, P. L. (Eds.), Fauna of Australia. Canberra: Australian Government Publishing Service, 1993, v. 2 A, p. $142-152$.

MALLMANN-FRANCO, M. T. O. Comparação anatômica e estrutura histológica das bolsas cloacais de oito espécies de Testudines. São Paulo: USP, 2002, 130p. Tese (Doutorado em Anatomia dos Animais Domésticos e Silvestres) - Programa de Pós-graduação em Anatomia dos Animais Domésticos e Silvestres, Universidade de São Paulo, São Paulo, 2002.

MALVASIO, A. Estudo anatômico do sistema urogenital e aspectos do sistema digestivo durante o desenvolvimento pós-natal de Trachemys dorbignyi (Duméril \& Bibron, 1835) (Reptilia, Testudines, Emydidae). São Paulo: USP, 1996. 68p. Dissertação (Mestrado em Zoologia) - Programa de Pós-graduação em Zoologia, Instituto de Ciências Biológicas, Universidade de São Paulo, São Paulo, 1996.

MESSER, H. M. An introduction to vertebrate anatomy. New York: The Mamillan, 1947.

MÉTRAILLER, S. Note sur lélevage et la reproduction de la matamata, Chelus fimbriatus (Schneider, 1783). France, Manouria, v.6, n.20, p.35-39. Set, 2003.

MONTAGNA, W. Comparative anatomy. New York: Jonh Wiley e Sons, 1959. 
ROSA, M.A. et al. Anatomia da cloaca de algumas espécies de Pleurodira (Reptilia:

Testudines). PUBVET, Londrina, V. 5, N. 11, Ed. 158, Art. 1070, 2011.

OLIVEIRA, C. A.; SILVA, R. M.; SANTOS, M. M.; MAHECHA, G. A. Location of the ureteral openings in the cloacas of tinamous, some Ratite birds, and crocodilians: a primitive character. Journal of Morphology, v.260, n.2, p. 234-246, New York, May. 2004.

PETERSON, C. C.; GREENSHIELDS, D. Negative test for cloacal drinking in a semi-aquatic turtle (Trachemys scripta), with comments on the functions of cloacal bursae. Journal of experimental zoology, United States, v.290, n.3, p.247-254, Aug. 2001.

POUGH, F. H.; JANIS, M. C.; HEISER, B. J. A vida dos vertebrados. 3ed. São Paulo: Atheneu, 2003.

PRITCHARD, P. C. H.; TREBBAU, P. The turtles of Venezuela. n.2 Venezuela: Society for the study of Amphibians and Reptiles, 1984.

ROMER, A. S.; PARSONS, T. S. Anatomia comparada dos vertebrados. São Paulo: Atheneu, 1985.

WEICHERT, C. K. Anatomy of chordates. New York: McGraw-Hill Book, 1951. 